\title{
General tensor Lagrangians from the gravitational Higgs mechanism
}

\author{
Durmuş A Demir ${ }^{1}$ and N K Pak ${ }^{2}$ \\ ${ }^{1}$ Department of Physics, Izmir Institute of Technology, TR35430 Izmir, Turkey \\ 2 Department of Physics, Middle East Technical University, TR06530 Ankara, Turkey
}

Received 21 November 2008, in final form 9 March 2009

Published 29 April 2009

Online at stacks.iop.org/CQG/26/105018

\begin{abstract}
The gravitational Higgs mechanism proposed by 't Hooft in arXiv:0708.3184 involves the spacetime metric $g_{\mu \nu}$ as well as the induced metric $\bar{g}_{\mu \nu} \propto$ $\eta_{a b} \partial_{\mu} \phi^{a} \partial_{\nu} \phi^{b}$ where $\phi^{a}(a=0, \ldots, 3)$, as we call it, break all four diffeomorphisms spontaneously via the vacuum expectation values $\left\langle\phi^{a}\right\rangle \propto x^{a}$. In this framework, we construct and analyze the most general action density in terms of various invariants involving the curvature tensors, connexion coefficients, and the contractions and the determinants of the two metric fields. We show that this action admits a consistent expansion about the flat background such that the resulting Lagrangian possesses several novel features not found in the linearized Einstein-Hilbert Lagrangian with FierzPauli mass term (LEHL-FP): (i) its kinetic part generalizes that of LELHL-FP by weighing the corresponding structures with certain coefficients generated by invariants, (ii) the entire Lagrangian is ghost- and tachyon-free for mass terms not necessarily in the Fierz-Pauli form, and, (iii) a consistent mass term is generated with no apparent need to higher derivative couplings.
\end{abstract}

PACS numbers: $04.00 .00,04.50 . \mathrm{Kd}, 11.30 . \mathrm{Qc}$

\section{Introduction and motivation}

In general, massive fields with spin $s \geqslant 1$ possess $2 s-1$ longitudinal components not found in their massless limit. These extra components directly couple to the conserved currents, and their effects do not necessarily disappear in the limit of vanishing mass. Therefore, there arises a discontinuity in the field's mass, and it renders the associated scattering amplitudes unphysical. This phenomenon is known to occur in non-Abelian gauge theories [1] as well as (the linearized) gravity [2]. Indeed, the linearized Einstein-Hilbert action, linearized about the flat spacetime in metric perturbations,

$$
h_{\mu \nu} \equiv g_{\mu \nu}-\eta_{\mu \nu}
$$


admits a mass term of the form

$$
\mathcal{L}_{\text {mass }}=-\frac{1}{4} M_{P l}^{2} m_{g}^{2}\left(h_{\alpha \beta} h^{\alpha \beta}-\zeta h_{\alpha}^{\alpha} h_{\beta}^{\beta}\right)
$$

where, $\zeta=1$ strictly, for the tensor theory to be ghost-free [3]. In other words, it is only and only for $\zeta=1$, the trace mode $h \equiv h_{\alpha}^{\alpha}$, which is a ghost as it possesses negative energy [4], decouples from the rest. As mentioned before, this tensor theory is discontinuous as $m_{g} \rightarrow 0$ [2].

A highly important feature of the Fierz-Pauli mass term (2), worthy of emphasizing here, is that it holds only in the linearized scheme. In other words, this very structure does not admit any nonlinear completion obeying general covariance. This immediately follows from the fact that, among the geometrical quantities pertaining to the spacetime manifold, there is no source, other than the determinant of the metric $\operatorname{det}\left(g_{\mu \nu}\right)$, for a non-derivative structure like the Fierz-Pauli mass term. However, one immediately runs into difficulties while trying to generate (2), if $\operatorname{det}\left(g_{\mu \nu}\right)$ is the only source. Indeed, a likely choice would be to augment Einstein-Hilbert action by vacuum energy contribution, $\sqrt{-\operatorname{det}\left(g_{\mu \nu}\right)} V_{v a c}$. However, this energy component cannot generate the graviton mass term correctly since, in the first place, background geometry is wrong and, secondly, the quadratic part of the linearized $\sqrt{-\operatorname{det}\left(g_{\mu \nu}\right)}$ yields (2) with $\zeta=1 / 2$ not $\zeta=1$. As a step further, one can imagine including higher powers of $\sqrt{-\operatorname{det}\left(g_{\mu \nu}\right)}$ for generating (2). However, this is simply impossible in general relativity since $\operatorname{det}\left(g_{\mu \nu}\right)$ is a scalar density and the general covariance gets blatantly broken unless some other scalar density (the determinant of some tensor field different than the metric) is appropriately incorporated into the action.

For a resolution of these problems (as reviewed in detail in [5]), it is considered convenient to start with an analysis of the mass discontinuity. This problem is overcome, in non-Abelian gauge theories, via the Higgs mechanism through which the gauge field develops requisite longitudinal component by swallowing the Goldstone boson generated by the spontaneous breakdown of the gauge symmetry. The system, as a whole, consists not only of the gauge field but also of the scalars, so that number of degrees of freedom remain unchanged as the state changes from symmetric to broken phase, and vice versa. In analogy with non-Abelian gauge theories, recently 't Hooft [6] (see also the previous work [7] and references therein), followed by $[8,9]$, proposed a similar mechanism for gravity in which graviton acquires mass via the spontaneous breakdown of the diffeomorphism invariance (see the earlier works [10-12, 13] for variant approaches).

In essence, what 't Hooft suggests is to introduce 'scalar coordinates' $\phi^{a}(x)(a=$ $0,1,2,3)$ which are functions of the 'vector coordinates' $x^{\mu}(\mu=0,1,2,3)$ of the spacetime (in the spirit of manifold structures [14] utilized for spacetime compactification). Nonvanishing vacuum expectation values (VEVs) of these scalars define a 'preference gauge', more precisely a 'preference frame' in which diffeomorphism invariance is spontaneously broken, whence graviton acquires a nonvanishing mass [6]. This mass generation process, compared to gauge theories, is complicated by the fact that the number of the scalars eaten is higher than the number of longitudinal degrees of freedom that should be generated. In other words, nonunitary degrees of freedom must be eliminated to have a physically sensible massive graviton. This point, in the framework of $[6,9]$, turns out to require higher derivative couplings in the action, in order to provide additional structures to eliminate the non-unitary modes. One important aspect of the present work will be to show that, elimination of the non-unitary modes does not necessarily necessitate such higher derivative terms.

By imposing the invariance under the shifts [6]

$$
\phi^{a} \rightarrow \phi^{a}+c^{a}
$$


it is automatically guaranteed that $(i) \phi^{a}$ cannot have non-derivative interactions such as a mass term, (ii) $\phi^{a}$ can interact only gravitationally via their kinetic terms, and finally (iii) all the effects of scalar coordinates can be encoded into the 'induced metric'

$$
\bar{g}_{\mu \nu}=\frac{1}{M^{4}} \eta_{a b} \nabla_{\mu} \phi^{a} \nabla_{\nu} \phi^{b}
$$

which is made dimensionless by rescaling its right-hand side by $M^{4}, M$ being a mass scale related to the VEV of the scalars $\phi^{a}$. At this point, for definiteness and clarity, it proves useful to dwell on the meanings and implications of the 'metric fields' to be used throughout the text:

- The 'metric' fields $g_{\mu \nu}$ and $\bar{g}_{\mu \nu}$ are actually spin-2 tensor fields. In the technical sense, they are not 'metric' fields. In fact, the former encodes the geometry (gravitational field) while the latter sets the background geometry with respect to which one studies the dynamics of spin-2 excitations $h_{\mu \nu}$ via (1). The true metric in this whole setup is the flat Minkowski metric $\eta_{\mu \nu}$. In spirit, the setup mimics that of bimetric gravity [16].

- The $\bar{g}_{\mu \nu}$, induced by the scalars $\phi^{a}$, is a tensor field that plays a role similar to that of the Higgs field in the spontaneously broken gauge theories. It equals $\eta_{\mu \nu}$ when all the four diffeomorphisms are broken spontaneously, and this sets the background geometry.

- The internal metric of the scalars is the flat Minkowski metric $\eta_{a b}$, not $\delta_{a b}$. In fact, structure of the induced metric in (4) parallels the decomposition $g_{\mu \nu}=\eta_{a b} e_{\mu}^{a} e_{v}^{b}$ so that the two metrics are related by the exchange of the vierbein $e_{\mu}^{a}$ and the gradient of the scalars $\nabla_{\mu} \phi^{a}$.

These observations reveal the distinctions among $g_{\mu \nu}, \bar{g}_{\mu \nu}$ and $\eta_{\mu \nu}$, though they will all be called 'metric' in what follows. The $g_{\mu \nu}$ and $\bar{g}_{\mu \nu}$ will be treated as two coexisting metric fields, though the latter reduces to $\eta_{\mu \nu}$ upon spontaneous diffeomorphism breaking.

In this work, we make use of the coexisting $\bar{g}_{\mu \nu}$ and $g_{\mu \nu}$ fields to write down the most general action density, and show that it admits a consistent expansion about the flat background such that the resulting Lagrangian owns several novel features not found in the linearized Einstein-Hilbert Lagrangian with Fierz-Pauli mass term (LEHL-FP): $(i)$ its kinetic part generalizes that of LELHL-FP by weighing the corresponding structures with generic coefficients formed by those of the invariants present in the action, (ii) the total Lagrangian qualifies to be ghost-and tachyon-free even for $\zeta \neq 1$ provided that the Lagrangian parameters satisfy certain consistency relations, and finally, (iii) a consistent mass term arises with no apparent need to higher derivative couplings.

The rest of the work is organized as follows. In section 2 below, we construct the action density after determining exhaustively the invariants made out of metric tensors, curvature tensors, and connexion coefficients. Also in this section, we derive the linearized action, determine the conditions on model parameters, and elaborate upon the generalized nature of the action by comparing it with the LEHL-FP framework, in regard to various extra structures not found in LEHL-FP setup. In section 3 we summarize our main findings, and conclude.

\section{The action}

For determining the most general action density, it proves useful to first prepare an inventory of the invariants. The non-derivative invariants are those constructed out of the metric fields, $\bar{g}_{\mu \nu}$ and $g_{\mu \nu}$. In this class, there naturally arise two fundamental invariants

$$
\mathrm{K}=g_{\mu \nu} \bar{g}^{\mu \nu}, \quad \mathrm{D}=\frac{\operatorname{det}\left(\bar{g}_{\mu \nu}\right)}{\operatorname{det}\left(g_{\mu \nu}\right)}
$$


where $\mathrm{K}$ is related to the kinetic term of the $\phi^{a}$, and the $\mathrm{D}$ to the ratio of the determinants of the two metrics. It is clear that $\mathrm{K}$ necessarily embodies a ghost state, since the scalar fields $\phi^{a}$ possess an indefinite metric $\left(\eta_{a b}\right.$ instead of $\left.\delta_{a b}\right)$. The invariants $\mathrm{K}$ and D are also invariant under the shift transformation (3). In fact, any function of them are also shift-invariant, and hence, they contribute to the action density via generic functions of the form $V_{1}(\mathrm{~K}), V_{2}(\mathrm{D})$ and $V_{3}(1 / \mathrm{D})$. These functions, as they stand, serve as shift-invariant 'potentials' for the metric field.

The derivative invariants, that is, those invariants which involve derivatives of the metric fields consist of a number of structures constructed from the curvature tensors and the connexion coefficients. Concerning the former, one readily finds two invariants

$$
\mathrm{R}_{1}=\bar{g}^{\mu \nu} g^{\alpha \beta} \mathcal{R}_{\mu \alpha \nu \beta}, \quad \mathrm{R}_{2}=\bar{g}^{\mu \nu} \bar{g}^{\alpha \beta} \mathcal{R}_{\mu \alpha \nu \beta}
$$

each of which dynamically differing from the usual Ricci scalar $\mathcal{R} \equiv g^{\mu \nu} g^{\alpha \beta} \mathcal{R}_{\mu \alpha \nu \beta}$. Not surprisingly, these are not the only curvature invariants since, being a metric tensor, the induced metric $\bar{g}_{\mu \nu}$ itself generates novel structures paralleling those generated by $g_{\mu \nu}$. To this end, in the spirit of constructing Lévi-Civitá connexion $\Gamma$ from the metric tensor $g_{\mu \nu}$, one can construct a different connexion

$$
\bar{\Gamma}_{\mu \nu}^{\lambda}=\frac{1}{2} \widehat{\bar{g}}^{\lambda \rho}\left(\partial_{\mu} \bar{g}_{\nu \rho}+\partial_{\nu} \bar{g}_{\rho \mu}-\partial_{\rho} \bar{g}_{\mu \nu}\right)
$$

based on $\bar{g}_{\mu \nu}$, assuming that it is invertible. Here, $\widehat{\bar{g}}^{\lambda \rho}$ is the matrix inverse of the induced metric $\bar{g}^{\lambda \rho}$, that is, $\widehat{\bar{g}}_{\lambda \rho} \bar{g}^{\rho \gamma}=\delta_{\lambda}^{\gamma}$. It is worth emphasizing again, that $\widehat{\bar{g}}^{\lambda \rho} \neq \bar{g}^{\lambda \rho} \equiv g^{\lambda \kappa} \bar{g}_{\kappa \theta} g^{\theta \rho}$. Needless to say, $\bar{\Gamma}_{\mu \nu}^{\lambda}$ is compatible with $\bar{g}_{\mu \nu}$ in full analogy with the compatibility of the $\Gamma_{\mu \nu}^{\lambda}$ with the $g_{\mu \nu}$.

As with the connexion $\Gamma_{\mu \nu}^{\lambda}$, the new connexion $\bar{\Gamma}_{\mu \nu}^{\lambda}$ also generates its Riemann tensor $\overline{\mathcal{R}}_{\mu \alpha \nu \beta}$ from which, similar to (6), one constructs the curvature invariants

$$
\overline{\mathrm{R}}_{1}=g^{\mu \nu} \bar{g}^{\alpha \beta} \overline{\mathcal{R}}_{\mu \alpha \nu \beta}, \quad \overline{\mathrm{R}}_{2}=g^{\mu \nu} g^{\alpha \beta} \overline{\mathcal{R}}_{\mu \alpha \nu \beta}
$$

in addition to the Ricci scalar $\overline{\mathcal{R}} \equiv \bar{g}^{\mu \nu} \bar{g}^{\alpha \beta} \overline{\mathcal{R}}_{\mu \alpha \nu \beta}$.

Apart from (6) and (8) generated by the curvature tensors of $g_{\mu \nu}$ and $\bar{g}_{\mu \nu}$, there exist extra invariants generated by the connexion coefficients. Indeed, the difference

$$
\mathcal{F}_{\mu \nu}^{\lambda}=\Gamma_{\mu \nu}^{\lambda}-\bar{\Gamma}_{\mu \nu}^{\lambda}
$$

is a rank $(1,2)$ tensor, and its contractions give rise to additional derivative invariants independent of the curvature tensors [16]. Obviously, all the invariants stemming from this tensor field necessarily involve even occurrences of $\mathcal{F}_{\mu \nu}^{\lambda}$. In fact, up to the quadratic order, possible invariants read as

$$
\begin{array}{rlrl}
\mathrm{C}_{1} & =g^{\mu \nu} \mathcal{F}_{\alpha \mu}^{\alpha} \mathcal{F}_{\beta \nu}^{\beta}, & \mathrm{C}_{2} & =g^{\mu \nu} \mathcal{F}_{\beta \mu}^{\alpha} \mathcal{F}_{\alpha \nu}^{\beta} \\
\mathrm{C}_{3} & =g^{\mu \nu} \mathcal{F}_{\alpha \beta}^{\alpha} \mathcal{F}_{\mu \nu}^{\beta}, & \mathrm{C}_{4} & =g^{\mu \nu} g^{\alpha \beta} g_{\lambda \rho} \mathcal{F}_{\alpha \mu}^{\lambda} \mathcal{F}_{\beta \nu}^{\rho} \\
\overline{\mathrm{C}}_{1} & =\bar{g}^{\mu v} \mathcal{F}_{\alpha \mu}^{\alpha} \mathcal{F}_{\beta \nu}^{\beta}, & \overline{\mathrm{C}}_{2}=\bar{g}^{\mu \nu} \mathcal{F}_{\beta \mu}^{\alpha} \mathcal{F}_{\alpha \nu}^{\beta} \\
\overline{\mathrm{C}}_{3}=\bar{g}^{\mu \nu} \mathcal{F}_{\alpha \beta}^{\alpha} \mathcal{F}_{\mu \nu}^{\beta}, & \overline{\mathrm{C}}_{4}=\bar{g}^{\mu \nu} g^{\alpha \beta} g_{\lambda \rho} \mathcal{F}_{\alpha \mu}^{\lambda} \mathcal{F}_{\beta \nu}^{\rho} \\
\overline{\mathrm{C}}_{5}=\bar{g}^{\mu \nu} \bar{g}^{\alpha \beta} g_{\lambda \rho} \mathcal{F}_{\alpha \mu}^{\lambda} \mathcal{F}_{\beta \nu}^{\rho}, & \overline{\mathrm{C}}_{6}=\bar{g}^{\mu \nu} \bar{g}^{\alpha \beta} \bar{g}_{\lambda \rho} \mathcal{F}_{\alpha \mu}^{\lambda} \mathcal{F}_{\beta \nu}^{\rho}
\end{array}
$$

Note that in these invariants indices on $\mathcal{F}_{\mu \nu}^{\lambda}$ are kept as in (9), with no further lowering or raising operations. 
Having determined all possible invariants in the presence of two metric fields, a general action integral can be written as

$$
\begin{aligned}
S_{G}=\frac{1}{2} M_{P l}^{2} \int & \mathrm{d}^{4} x \sqrt{-\operatorname{det}\left(g_{\mu \nu}\right)}\left\{\mathcal{R}+\bar{a} \overline{\mathcal{R}}+a_{1} \mathrm{R}_{1}+a_{2} \mathrm{R}_{2}+\bar{a}_{1} \overline{\mathrm{R}}_{1}+\bar{a}_{2} \overline{\mathrm{R}}_{2}\right. \\
& \left.+\sum_{i=1}^{4} c_{i} \mathrm{C}_{i}+\sum_{j=1}^{6} \bar{c}_{j} \overline{\mathrm{C}}_{j}+V_{1}(\mathrm{~K})+V_{2}(\mathrm{D})+V_{3}(1 / \mathrm{D})\right\}
\end{aligned}
$$

wherein the derivative invariants with higher mass dimension ( $\operatorname{such}$ as $\mathcal{R}^{n}, \mathrm{C}_{i}^{2}, \mathrm{R}_{i}^{n}$ with $n \geqslant 2$ ) are ignored. Therefore, $\bar{a}, a_{i}, \bar{a}_{i}, c_{i}$ and $\bar{c}_{i}$ are all dimensionless constants. In spite of this restricted structure of the sector of derivative invariants, the sector of the non-derivative invariants, represented by the 'potentials' $V_{1,2,3}$, is kept as general as possible to cope with the constraints that can be faced with while inducing a consistent graviton mass term.

The action density in (11) incorporates, in the geometrical sector, all possible invariants within the aforementioned limits. Obviously, this action provides a more general framework than those in the existing literature, as the original proposal of 't Hooft [6], and its refinements $[8,9]$ involve only $\mathcal{R}$ and $V_{1}(\mathrm{~K})$ contributions. The extra structures, as will be shown in the next section, give rise to novel features in relation to structuring of the background geometry, canceling the tadpoles, and killing the ghosts.

\section{Higgsing gravity}

The general covariance guarantees that physical quantities are independent of the choice of the coordinates. For instance, invariance of the Einstein-Hilbert action under the infinitesimal coordinate transformations ( $\epsilon$ being infinitesimal)

$$
x^{\mu} \rightarrow x^{\mu}-\epsilon^{\mu}(x)
$$

reflects itself in the conservation law expressed by the contracted differential Bianchi identity. This coordinate transformation or 'gauge transformation' gives rise to the diffeomorphisms

$$
\delta g_{\mu \nu}=\nabla_{\mu} \epsilon_{\nu}+\nabla_{\nu} \epsilon_{\mu}, \quad \delta \phi^{a}=\nabla_{\mu} \phi^{a} \epsilon^{\mu}
$$

as dictated by variations of the metric $g_{\mu \nu}$, and the scalars $\phi^{a}$ under general coordinate transformations. This very reparametrization invariance is the fundamental gauge symmetry of the action (11) in that its status - exact or broken - determines whether or not there exist massive excitations in the spectrum. Before turning our attention to the broken symmetry case, which is the main aim of this work, we first briefly discuss the case of exact diffeomorphism invariance, corresponding to the massless graviton, for completeness.

\subsection{Massless graviton}

In massless phase, reparametrization invariance is exact. The vacuum configuration

$$
\left\langle g_{\mu \nu}\right\rangle=\eta_{\mu \nu}, \quad\left\langle\phi^{a}\right\rangle=0
$$

for which $\left\langle\bar{g}_{\mu \nu}\right\rangle=0$ obviously, can be sustained, as all the curvature invariants vanish trivially, if the potential functions satisfy the constraint

$$
V_{1}(0)+V_{2}(0)+V_{3}(\infty)=0 \text {. }
$$

There is a subtlety involving the invariants $\mathrm{C}_{i}$ and $\overline{\mathrm{C}}_{i}$. That is while $\Gamma_{\mu \nu}^{\lambda}=0$ trivially for strictly flat metric, the connexion $\bar{\Gamma}_{\mu \nu}^{\lambda}$ of the induced metric appears to have $\frac{0}{0}$ indeterminacy. 
Nonetheless, this indeterminacy does not mean that $\bar{\Gamma}_{\mu \nu}^{\lambda}$ diverges. In fact, with an appropriate regularization procedure, for example, $\left\langle\phi^{a}\right\rangle=\delta_{1} x^{a}+\delta_{2}^{b} x_{b} x^{a}$ with $\delta_{2}^{a} \ll \delta_{1}$ and $\delta_{2}^{a} \rightarrow 0$, it can be adjusted to vanish. Consequently, the invariants $\mathrm{C}_{i}$ and $\overline{\mathrm{C}}_{i}$ all vanish in the symmetric vacuum (14) since $\left\langle\mathcal{F}_{\mu \nu}^{\lambda}\right\rangle=0$ therein.

The excitations about the vacuum configuration (14) involve two propagating degrees of freedom pertaining to $h_{\mu \nu}$ and four associated with $\phi^{a}$ since all the diffeomorhisms in (13) are exact. These six degrees of freedom constitute the symmetric phase of the fields and the interactions encoded in (11).

We have to reiterate that this regularization procedure affects only the massless case. That it is, once $\bar{\Gamma}_{\mu \nu}^{\lambda}$ is regulated to vanish when $\bar{g}_{\mu \nu} \rightarrow 0$, as long as the constraint in (15) is satisfied, one realizes the massless gravity limit smoothly. For the massive case, which is at the focus of the present work, this subtlety is irrelevant, however.

\subsection{Massive graviton}

In the massive phase, reparametrization invariance is spontaneously broken. Indeed, the vacuum configuration ( $M$ being the mass scale appearing in (4))

$$
\left\langle g_{\mu \nu}\right\rangle=\eta_{\mu \nu}, \quad\left\langle\phi^{a}\right\rangle=M^{2} x^{a},
$$

breaks the diffeomorphism invariance spontaneously and thus, as in gauge theories, defines a 'preference frame' such that all temporal and spatial diffeomorphisms are broken by imposing

$$
\delta \phi^{a}=0
$$

in (13). This gauge fixing forces all four scalar fields $\phi^{a}$ to remain stuck to their VEVs in (16), leaving behind no scalar fluctuations to propagate. Expectedly, again in complete similarity to gauge theories, this gauge fixing procedure automatically renders all 10 components of $h_{\mu \nu}$ physical. However, a massive tensor field can have only 5 propagating modes, and thus, the 5 extra components should be eliminated by the dynamics encoded in (11).

Under the gauge fixing (17), the building blocks of the invariants in (11) can be systematically expanded about (16) as follows:

(1) The scalars $\phi^{a}$ are fixed to their VEVs: $\phi^{a}=\left\langle\phi^{a}\right\rangle=M^{2} x^{a}$.

(2) The quantities involving the spacetime metric $g_{\mu \nu}$ we expanded up to quadratic order as

$$
\begin{aligned}
& g_{\mu \nu}=\eta_{\mu \nu}+h_{\mu \nu}, \\
& g^{\mu \nu}=\eta^{\mu \nu}-h^{\mu \nu}+h^{\mu \alpha} h_{\alpha}^{\nu}+\mathcal{O}\left(h^{3}\right), \\
& -\operatorname{det}\left(g_{\mu \nu}\right)=1+h+\frac{1}{2} h^{2}-\frac{1}{2} h^{\alpha \beta} h_{\alpha \beta}+\mathcal{O}\left(h^{3}\right), \\
& \Gamma_{\mu \nu}^{\lambda}=\frac{1}{2}\left(\eta^{\lambda \rho}-h^{\lambda \rho}\right)\left(\partial_{\mu} h_{\nu \rho}+\partial_{\nu} h_{\rho \mu}-\partial_{\rho} h_{\mu \nu}\right)+\mathcal{O}\left(h^{3}\right) .
\end{aligned}
$$

(3) The quantities related to the induced metric are expanded as

$$
\begin{aligned}
& \bar{g}_{\mu \nu}=\eta_{\mu \nu}, \\
& \bar{g}^{\mu \nu}=\eta^{\mu \nu}-2 h^{\mu \nu}+3 h^{\mu \alpha} h_{\alpha}^{v}+\mathcal{O}\left(h^{3}\right), \\
& -\operatorname{det}\left(\bar{g}_{\mu \nu}\right)=1, \\
& \bar{\Gamma}_{\mu \nu}^{\lambda}=0 .
\end{aligned}
$$

In addition, one has $\widehat{\bar{g}}_{\mu \nu}=\eta_{\mu \nu}$, and since $\widehat{\bar{g}}^{\mu \nu}$ is the matrix inverse of $\bar{g}^{\mu \nu}$, it is immediately found that $\widehat{\bar{g}}^{\mu \nu}=\eta^{\mu \nu}$.

Having (17), (18) and (19) at hand, one can readily expand the action density in (11) about the vacuum configuration (16) to obtain the $h_{\mu \nu}$ Lagrangian. To begin with, one notes 
that $\overline{\mathcal{R}}, \overline{\mathrm{R}}_{1}, \overline{\mathrm{R}}_{2}$ all vanish identically, as follows from (19). The rest of the derivative invariants give rise to the action density

$$
\begin{aligned}
-\frac{M_{P l}^{2}}{2}\left[\left(1+a_{1}\right.\right. & \left.+a_{2}\right)\left(\partial_{\lambda} \partial_{\rho} h^{\lambda \rho}-\square h\right)+\frac{\widehat{a}}{4} \partial_{\lambda} h^{\alpha \beta} \partial^{\lambda} h_{\alpha \beta} \\
+ & \left.\frac{\widehat{b}}{2} \partial_{\lambda} h^{\lambda \alpha} \partial^{\rho} h_{\rho \alpha}+\frac{\widehat{c}}{2} \partial_{\lambda} h \partial_{\rho} h^{\lambda \rho}+\frac{\widehat{d}}{4} \partial_{\lambda} h \partial^{\lambda} h\right]
\end{aligned}
$$

whose first line, linear in $h_{\mu \nu}$, can obviously be discarded away since it is a total divergence. The quadratic terms in the second line form the kinetic part of the total $h_{\mu \nu}$ action. The hatted coefficients herein read in terms of the original ones in (11) as follows

$$
\begin{aligned}
& \widehat{a}=1+3 a_{1}+5 a_{2}+c_{2}+\bar{c}_{2}-3\left(c_{4}+\bar{c}_{4}+\bar{c}_{5}+\bar{c}_{6}\right) \\
& \widehat{b}=-1-3 a_{1}-5 a_{2}+c_{4}+\bar{c}_{4}+\bar{c}_{5}+\bar{c}_{6}-c_{2}-\bar{c}_{2} \\
& \widehat{c}=1+2 a_{1}+3 a_{2}-\frac{1}{2}\left(c_{3}+\bar{c}_{3}\right) \\
& \widehat{d}=-1-a_{1}-a_{2}-c_{1}-\bar{c}_{1} .
\end{aligned}
$$

In each coefficient, the right-hand side starts with \pm 1 which is what would be found within LEHL-FP formalism, and the additional terms represent the deviations due to the curvature invariants occurring in the presence of $\bar{g}_{\mu \nu}$.

The non-derivative part of the action originates from the 'potentials' $V_{1,2,3}$ in (11). Expanding them up to quadratic order by using (17), (18) and (19), the action density turns out to be $M_{P l}^{2} / 2$ times

$$
\begin{aligned}
V_{1}(4)+V_{2}(1)+ & V_{3}(1)+\left[V_{3}^{\prime}(1)-V_{1}^{\prime}(4)-V_{2}^{\prime}(1)+\frac{1}{2}\left(V_{1}(4)+V_{2}(1)+V_{3}(1)\right)\right] h \\
& +\left[\frac{1}{2}\left(V_{3}^{\prime}(1)-V_{1}^{\prime}(4)-V_{2}^{\prime}(1)\right)+\frac{1}{8}\left(V_{1}(4)+V_{2}(1)+V_{3}(1)\right)\right. \\
& \left.+\frac{1}{2}\left(V_{2}^{\prime}(1)+V_{3}^{\prime}(1)+V_{1}^{\prime \prime}(4)+V_{2}^{\prime \prime}(1)+V_{3}^{\prime \prime}(1)\right)\right] h^{2} \\
& +\left[-\frac{1}{4}\left(V_{1}(4)+V_{2}(1)+V_{3}(1)\right)-\frac{1}{2}\left(V_{3}^{\prime}(1)-V_{1}^{\prime}(4)-V_{2}^{\prime}(1)\right)\right. \\
& \left.+\frac{1}{2} V_{1}^{\prime}(4)\right] h^{\alpha \beta} h_{\alpha \beta}
\end{aligned}
$$

where primes on $V_{i}$ 's denote derivatives with respect to their arguments. This action density is subject to certain consistency conditions beyond (15) found in the symmetric phase. First, for the entire procedure to be consistent, the background geometry must be flat Minkowski, that is, the total vacuum energy must vanish

$$
V_{1}(4)+V_{2}(1)+V_{3}(1)=0 \text {. }
$$

Next, in (22) the terms linear in $h$ must also vanish

$$
V_{3}^{\prime}(1)-V_{1}^{\prime}(4)-V_{2}^{\prime}(1)=0
$$

as otherwise classical field configuration gets destabilized (or tadpoles are generated in quantized theory).

Combining the kinetic part in (20) with the remnant of (22) after imposing (23) and (24), the total $h_{\mu \nu}$ action takes the form

$$
\begin{aligned}
S_{L}=-\frac{M_{P l}^{2}}{2} \int & \mathrm{d}^{4} x\left[\frac{\widehat{a}}{4} \partial_{\lambda} h^{\alpha \beta} \partial^{\lambda} h_{\alpha \beta}+\frac{\widehat{b}}{2} \partial_{\lambda} h^{\lambda \alpha} \partial^{\rho} h_{\rho \alpha}+\frac{\widehat{c}}{2} \partial_{\lambda} h \partial_{\rho} h^{\lambda \rho}\right. \\
& \left.+\frac{\widehat{d}}{4} \partial_{\lambda} h \partial^{\lambda} h+\frac{1}{4} m_{g}^{2}\left(h^{\alpha \beta} h_{\alpha \beta}-\zeta h^{2}\right)\right]
\end{aligned}
$$


where the mass term, taken to be precisely in the form of (2), involves the 'graviton mass'

$$
m_{g}^{2}=-2 V_{1}^{\prime}(4)
$$

as well as

$$
\zeta=-\frac{1}{V_{1}^{\prime}(4)}\left(V_{1}^{\prime}(4)+2 V_{2}^{\prime}(1)+V_{1}^{\prime \prime}(4)+V_{2}^{\prime \prime}(1)+V_{3}^{\prime \prime}(1)\right)
$$

where uses have been made of the conditions (23) and (24). These conditions, that the vacuum energy and tadpoles must vanish, impose strong fine-tuning constraints on the potentials $V_{i}$. Their violations destabilize the background geometry and render the whole procedure inconsistent. Apart from them, there arise additional constraints stemming from the $h_{\mu \nu}$ dynamics itself, as to be determined below.

The action (25), in form, embodies the most general quadratic-level Lagrangian for a symmetric tensor field. In fact, it is precisely the generic tensor theory setup studied in [15], which provides a detailed analysis of propagating modes and elimination of the ghosts and tachyons. Nonetheless, for the present analysis, it proves particularly useful to focus on the equations of motion themselves, especially for a clear view of the dynamics of the scalar ghost $h$. The equations of motion for $h_{\mu \nu}$, as originate from the extremization of (25), read as

$$
\begin{gathered}
\widehat{a} \square h_{\mu \nu}+\widehat{b}\left(\partial_{\mu} \partial_{\rho} h_{\nu}^{\rho}+\partial_{\nu} \partial_{\rho} h_{\mu}^{\rho}\right)+\widehat{c} \eta_{\mu \nu} \partial_{\rho} \partial_{\lambda} h^{\rho \lambda}+\widehat{c} \partial_{\mu} \partial_{\nu} h \\
+\widehat{d} \eta_{\mu \nu} \square h-m_{g}^{2}\left(h_{\mu \nu}-\zeta h \eta_{\mu \nu}\right)=0
\end{gathered}
$$

which can be mapped into dynamical equations of lower spin components by repeatedly applying contraction and divergence operations. This way, the trace component $h$ is found to obey

$$
b_{2} \square^{2} h+b_{1} m_{g}^{2} \square h+b_{0} m_{g}^{4} h=0
$$

wherein

$$
\begin{aligned}
& b_{2}=-\widehat{a}^{2}-2 \widehat{a}(\widehat{b}+\widehat{c}+2 \widehat{d})-6 \widehat{b} \widehat{d}+3 \widehat{c}^{2}, \\
& b_{1}=-2((1-2 \zeta) \widehat{a}+(1-3 \zeta) \widehat{b}+\widehat{c}+2 \widehat{d}), \\
& b_{0}=4 \zeta-1,
\end{aligned}
$$

which involve the model parameters in (21) at the order indicated by their subscripts.

The equations of motion for the vector component $\partial_{\mu} h^{\mu v}$ is

$$
\begin{gathered}
{\left[(-\widehat{a}-\widehat{b}) \square+m_{g}^{2}\right] \partial_{\mu} h^{\mu \nu}+\frac{1}{2 \widehat{b}+4 \widehat{c}}[(\widehat{a}(\widehat{b}+\widehat{c})-\widehat{b}(\widehat{c}-2 \widehat{d})) \square} \\
\left.-(\widehat{c}+(1-2 \zeta)) m_{g}^{2}\right] \partial^{v} h=0
\end{gathered}
$$

This is coupled to (29) via the gradient of $h$.

The equations of motion (29) and (31) reveal the unphysical degrees of freedom contained in $h_{\mu \nu}$. Indeed, as the first point to note, the trace field $h$ is clearly a ghost, and therefore, it should be prohibited to propagate. This is accomplished by requiring

$$
b_{2}=0, \quad b_{1}=0
$$

in (29). The main consequence of these conditions is that $h$ is eliminated from the 10 total degrees of freedom in $h_{\mu \nu}$ since, from (29), $h=0$ follows unambiguously (One might alternatively consider taking $b_{0}=0$ as this also satisfies (29). However, this choice does not eliminate $h$ from the spectrum; moreover, it gives rise to a ghosty graviton as it enforces $\zeta=1 / 4$.). 
Setting $h=0$ in (31) reveals that the vector $\partial_{\mu} h^{\mu \nu}$ is also a ghost, and its elimination from the spectrum requires

$$
\widehat{a}+\widehat{b}=0
$$

as a further condition for having a ghost-free tensor theory.

Consequently, the equations of motion (28), after eliminating scalar ghost $h$ and vector ghost $\partial_{\mu} h^{\mu \nu}$, take the form

$$
\begin{aligned}
& h=0, \\
& \partial_{\mu} h^{\mu v}=0, \\
& \left(\widehat{a} \square-m_{g}^{2}\right) h_{\mu \nu}=0,
\end{aligned}
$$

where, obviously, it is imperative to have

$$
\widehat{a}>0, \quad m_{g}^{2}>0
$$

for equations of motion (34) to describe a non-ghost, non-tachyonic, massive, free, spin-2 field. The wave equation for $h_{\mu \nu}$ can be put into conventional form by rescaling the action (25) by $1 / \widehat{a}$. Positivity of $m_{g}^{2}$, through (26), implies that $V_{1}^{\prime}(4)<0$. The main implication of this, recalling that $\mathrm{K}=g_{\mu \nu} \bar{g}^{\mu \nu}=4-h+\mathcal{O}\left(h^{2}\right)$, is that $V_{1}(\mathrm{~K})$ must obtain negative slope around $\mathrm{K}=4$ so as to invert the signature of $\bar{g}_{\mu \nu}$ controlled by $\eta_{a b}$. This switch of signature makes $m_{g}^{2}$ positive, or equivalently, the graviton non-tachyonic.

The equations of motion (34) hold only for free $h_{\mu \nu}$. The matter sector can be incorporated into geometrodynamics by augmenting the action (11) with

$$
\Delta S_{G}=\int \mathrm{d}^{4} x \sqrt{-\operatorname{det}\left(g_{\mu \nu}\right)} \mathcal{L}_{\text {matter }}(g, \bar{g}, \psi)
$$

where $\psi$ stands for matter fields, collectively. This add-on interaction causes the $h_{\mu \nu}$ action (25) to be extended by $-(1 / 2) h^{\mu \nu} T_{\mu \nu}^{\text {matter }}$. Consequently, unless the matter stress tensor $T_{\mu \nu}^{\text {matter }}$ possesses certain special features, all components of $h_{\mu \nu}$, excluding the vector ghost $\partial_{\mu} h^{\mu \nu}$, couple to and affected by $T_{\mu \nu}^{\text {matter }}$. This implies, in particular, that the equation of motion of $h$ (29) possesses an inhomogeneity involving the trace of $T_{\mu \nu}^{\text {matter }}$. If the matter sector is not conformal invariant, which indeed is not, it becomes impossible to eliminate $h$, or equivalently, to obtain $h=0$. This problem was already noticed by 't Hooft in [6], and a resolution was suggested: Similar to the potentials $V_{2,3}$, the matter Lagrangian should also depend on the metric tensor via the determinantal invariant D. More explicitly, the matter Lagrangian must have the specific structure

$$
\mathcal{L}_{\text {matter }}\left(g_{\mu \nu}, \bar{g}_{\mu \nu}, \psi\right) \equiv \mathcal{L}_{\text {matter }}\left(\mathrm{D}^{1 / 6} g_{\mu \nu}, \psi\right)
$$

so that the scalar ghost $h$ gets eliminated despite the presence of matter.

Having reached a physically sensible picture of massive graviton, at this stage it could be useful to perform a global analysis of the resulting constraints on the model parameters. Tabulated in table 1 are the constraints imposed by having ghost- and tachyon-free massive gravity. The implications or status of the constraints are shown for both the LEHL-FP and the present model. In the linearized Einstein-Hilbert action with Fierz-Pauli type mass term (the LEHL-FP framework), the parameters $\widehat{a}, \ldots, \widehat{d}$ take on rather specific values such that all the bounds and constraints are satisfied trivially (designated by the symbol $\sqrt{ }$ in the third column). The only exception is $\zeta$, namely $b_{1}=0$ requires $\zeta=1$ which is the unique value of $\zeta[9,15]$ for the Fierz-Pauli mass term defined in (2).

Concerning the model under investigation, constraints and resulting bounds on or relations among the model parameters are displayed in the fourth column of table 1. The fact that $\widehat{a}, \ldots, \widehat{d}$ deviate from LEHL-FP limit due to nonvanishing $a_{i}, c_{i}$ and $\bar{c}_{i}$ contributions, leaves 
important impact on parametric relations arising in response to bounds and constraints. In particular, none of the constraints (listed in the second column) is satisfied trivially; each is realized at the expense of imposing a further relation, which itself leads to the determination or bounding of a certain parameter in terms of the others. The constraints are not sufficient in number for a full determination of the model parameters. Nevertheless, various relations in the fourth column reflect the generalized nature of (25) with respect to the LEHL-FP framework. A highly important feature is that $\zeta$ is forced to have a specific relation to $\widehat{a}, \widehat{b}$ and $\widehat{c}$. However, as a direct consequence of the gravitational Higgs mechanism, the same parameter is related also to the potential functions $V_{i}$, at specific values of these arguments as depicted in (27). Therefore, elimination of the scalar ghost imposes a direct correlation between the derivative and non-derivative sectors in (11) by forcing $\zeta$ to be equal to

$$
\begin{aligned}
\zeta= & \frac{\widehat{a}^{2}-3 \widehat{a} \widehat{c}+3 \widehat{c}^{2}}{\widehat{a}^{2}} \\
= & \frac{1}{4\left(1+3 a_{1}+5 a_{2}+c_{2}+\bar{c}_{2}\right)^{2}}\left[4+12 a_{1}^{2}+28 a_{2}^{2}\right. \\
& +6 a_{1}\left(2+6 a_{2}-c_{3}-\bar{c}_{3}\right)+4\left(c_{2}+\bar{c}_{2}\right)\left(c_{2}+\bar{c}_{2}-1\right) \\
& +2 a_{2}\left(8+2\left(c_{2}+\bar{c}_{2}\right)-3\left(c_{3}+\bar{c}_{3}\right)\right) \\
& \left.+3\left(c_{3}+\bar{c}_{3}\right)\left(-2+c_{3}+\bar{c}_{3}+2\left(c_{2}+\bar{c}_{2}\right)\right)\right] \\
= & -\frac{1}{V_{1}^{\prime}(4)}\left(V_{1}^{\prime}(4)+2 V_{2}^{\prime}(1)+V_{1}^{\prime \prime}(4)+V_{2}^{\prime \prime}(1)+V_{3}^{\prime \prime}(1)\right)
\end{aligned}
$$

where use has been made of (21) and (33) in the second step. This equality can be used to eliminate one of the unknowns. For instance, it can be used to solve $c_{2}+\bar{c}_{2}$ in terms of $c_{3}+\bar{c}_{3}, a_{1}, a_{2}$ and the potentials in the second line. The solution, after replacing in the third row of table 1 , determines $c_{1}+\bar{c}_{1}$ in terms of $c_{3}+\bar{c}_{3}, a_{1}, a_{2}$ and the potentials. This, however, does not bring any important novelty in that $c_{1}+\bar{c}_{1}$ just gets expressed in terms of the potential functions instead of $c_{2}+\bar{c}_{2}$. Nonetheless, extraction of $c_{2}+\bar{c}_{2}$ from (38) gives some useful bounds in light of the constraint $\widehat{a}>0$ (implying $1+3 a_{1}+5 a_{2}+c_{2}+\bar{c}_{2}>0$ as shown in the fourth row of table 1). Indeed, one finds that

$$
1+3 a_{1}+5 a_{2}+c_{2}+\bar{c}_{2}=\frac{3}{4 \varpi+2}\left(2+4 a_{1}+6 a_{2}-c_{3}-\bar{c}_{3}\right)\left[1 \pm \sqrt{-\frac{1}{3}(4 \varpi+5)}\right]
$$

where

$$
-1-\varpi \equiv-\frac{1}{V_{1}^{\prime}(4)}\left(V_{1}^{\prime}(4)+2 V_{2}^{\prime}(1)+V_{1}^{\prime \prime}(4)+V_{2}^{\prime \prime}(1)+V_{3}^{\prime \prime}(1)\right)
$$

which equals the second line of (38). The \pm signs correspond to the two solutions of $c_{2}+\bar{c}_{2}$ as extracted from (38). This quantity can be guaranteed to be positive by various combinations of signs and magnitudes of the parameters at the right-hand side. On the other hand, the parameter $\varpi$ is bounded by

$$
\varpi<-\frac{5}{4}
$$

as follows from the terms in the radical sign in (39). This then gives rise to the constraint

$$
2 V_{2}^{\prime}(1)+V_{1}^{\prime \prime}(4)+V_{2}^{\prime \prime}(1)+V_{3}^{\prime \prime}(1)>\frac{5}{4}\left|V_{1}^{\prime}(4)\right|
$$

after using the inequality $V_{1}^{\prime}(4)<0$ for graviton to be non-tachyonic (as indicated in the fifth row of table 1). However, there is more than this. Indeed, after using (42) in the definition of $\zeta$ in (27), one arrives at the bound

$$
\zeta>\frac{1}{4}
$$


Table 1. Constraints on the model parameters for having a ghost- and tachyon-free massive graviton. The implication or status of each constraint is depicted for LEHL-FP (the third column) and the present model (the fourth column). The symbol $\sqrt{ }$ means that a given constraint is satisfied trivially (as happens for LEHL-FP for all constraints except for $b_{1}=0$ ) or upon the imposition of a condition which itself constrains or determines certain parameters in terms of the others (as happens for the present formalism in all cases).

\begin{tabular}{|c|c|c|c|}
\hline Equation & Relation & $\begin{array}{l}\text { LEHL-FP Model } \\
(\widehat{a}=-\widehat{b}=\widehat{c}=-\widehat{d}=1)\end{array}$ & $\begin{array}{l}\text { Present Model (equation (11) or } \\
\text { equation (25)) (see equation (21) } \\
\text { for parameters) }\end{array}$ \\
\hline Equation (33) & $\widehat{a}+\widehat{b}=0$ & $\sqrt{ }$ & $\begin{array}{l}\sqrt{ } \text { if } c_{4}+\bar{c}_{4}+\bar{c}_{5}+\bar{c}_{6}=0 \\
\sqrt{ } \text { if } \widehat{d}=-\left((\widehat{a}-\widehat{c})^{2}+2 \widehat{c}^{2}\right) / 2 \widehat{a}\end{array}$ \\
\hline Equation (32) & $b_{2}=0$ & $\sqrt{ }$ & $\left(\begin{array}{l}c_{1}+\bar{c}_{1}=\frac{1}{8\left(1+3 a_{1}+5 a_{2}+c_{2}+\bar{c}_{2}\right)} \\
\times\left[3\left(c_{3}+\bar{c}_{3}\right)^{2}-4\left(c_{3}+\bar{c}_{3}\right)\left(2+3 a_{1}+4 a_{2}\right.\right. \\
\left.-c_{2}-\bar{c}_{2}\right)+4\left(3 a_{1}^{2}+12 a_{1} a_{2}+12 a_{2}^{2}\right. \\
\left.\left.+\left(c_{2}+\bar{c}_{2}\right)^{2}+2\left(a_{2}-1\right)\left(c_{2}+\bar{c}_{2}\right)\right)\right]\end{array}\right)$ \\
\hline Equation (35) & $\widehat{a}>0$ & $\sqrt{ }$ & $\sqrt{ }$ if $1+3 a_{1}+5 a_{2}+c_{2}+\bar{c}_{2}>0$ \\
\hline Equation (35) & $m_{g}^{2}>0$ & $\sqrt{ }$ & $\sqrt{ }$ if $V_{1}^{\prime}(4)<0$ \\
\hline Equation (32) & $b_{1}=0$ & $\sqrt{ }$ if $\zeta=1$ & $\sqrt{ }$ if $\zeta=\left(\widehat{a}^{2}-3 \widehat{a} \widehat{c}+3 \widehat{c}^{2}\right) / \widehat{a}^{2}>\frac{1}{4}$ \\
\hline
\end{tabular}

which clearly shows that $\zeta$ is positive yet does not need to take its value preferred by the Fierz-Pauli mass term. This bound is indicated in the last row of table 1.

It is clear that the elimination of the scalar ghost does only put a bound on $\zeta$ as given in (43). For instance, there is no obligation to have one or all of the $V_{i}^{\prime \prime}$ to be nonzero. Indeed, they can all vanish without causing a problem, provided that $V_{2}^{\prime}(1)$ assumes an appropriate value to satisfy (42). In this sense, thanks to the inclusion of determinantal invariants, $V_{2}$ (D) and $V_{3}(1 / \mathrm{D})$ in (11), it becomes possible to induce a physically consistent graviton mass with no fundamental need to the higher derivative couplings. This is a novel feature not found in [9], wherein it is shown that the existence of higher derivative couplings are essential for eliminating the $h$.

\section{Conclusion}

In this work, by exploiting the coexistence of two metric fields $\bar{g}_{\mu \nu}$ and $g_{\mu \nu}$ in the gravitational Higgs mechanism proposed by 't Hooft [6], we have constructed and studied the most general action functional (11). The action involves both derivative (originating from the curvature tensors and the connexion coefficients) as well as non-derivative (originating from both $g_{\mu \nu}$ and $\bar{g}_{\mu \nu}$ ) invariants.

We have shown that the action density in (25) admits a consistent expansion about the flat background such that the resulting Lagrangian (25) possesses several novel features not found in the linearized Einstein-Hilbert Lagrangian with the Fierz-Pauli mass term. First of all, its kinetic part generalizes that of the LELHL-FP framework by weighing the corresponding structures with generic coefficients (21) generated by the invariants present in (11). Next, a ghost- and tachyon-free massive gravity theory arises, once the conditions in the table 1 are met. In particular, the absence of the ghosts and the tachyons does not require $\zeta=1$; it takes a general value shown in the fifth row of the table 1, provided that the constraint (38) is respected. 
It is true that the action in (11) contains various independent structures which come with independent coefficients. We have checked that one can eliminate several of these by making use of the relations stemming from the constraints tabled in the fourth column of the table 1. However, as the number of the constraints is fewer than the number of parameters, there are yet several free parameters left over in the scheme after eliminating as many of these as the constraints enable us to do. The parameters $c_{3}+\bar{c}_{3}, a_{1}, a_{2}$ and various potential functions remain as essentially free parameters (as long as (42) and the bounds in fourth and fifth rows of table 1 are satisfied).

Another important feature concerns the nature of the non-derivative invariants. The inclusion of the determinantal invariants facilitates generation of the graviton mass term with no apparent need to the higher derivative couplings. In other words, the potentials $V_{i}$ can have vanishing derivatives at the second and the higher orders, yet a physically meaningful graviton mass still arises, as shown in (38).

In the entire text the focus of our attention was on the massive gravity, only. However, this does not need to be so. Indeed, the action (25) does also describe of glueball dynamics in QCD after the replacements $M_{P l} \rightarrow \Lambda_{Q C D}$ and $m_{g} \sim 1 \mathrm{GeV}$. Therefore, generality of (25) can also provide useful tools for exploring the glueballs in QCD.

\section{Acknowledgments}

The research of NKP was supported by the Turkish Academy of Sciences through a membership research grant. The work of DD was supported by Alexander von HumboldtStiftung Friedrich Wilhelm Bessel-Forschungspreise and by the Turkish Academy of Sciences via GEBIP grant. DD is grateful for the hospitality of the Theory Group at DESY, Hamburg where this work was started.

\section{References}

[1] Vainshtein A I and Khriplovich I B 1971 Sov. J. Nucl. Phys. 13111 Vainshtein A 2006 Surveys High Energ. Phys. 205

[2] Iwasaki Y 1970 Phys. Rev. D 22255 van Dam H and Veltman M J G 1970 Nucl. Phys. B 22397 Zakharov V I 1970 JETP Lett. 12312 Zakharov V I 1970 Pisma Zh. Eksp. Teor. Fiz. 12447

[3] Fierz M and Pauli W 1939 Proc. Roy. Soc. Lond. A 173211

[4] Boulware D G and Deser S 1972 Phys. Rev. D 63368

[5] Rubakov V A and Tinyakov P G 2008 Phys. Usp. 51759 (arXiv:0802.4379 [hep-th])

[6] 't Hooft G 2007 arXiv:0708.3184 [hep-th]

[7] Arkani-Hamed N, Georgi H and Schwartz M D 2003 Ann. Phys. 30596 (arXiv:hep-th/0210184)

[8] Kakushadze Z 2008 Int. J. Mod. Phys. A 231581 (arXiv:0709.1673 [hep-th]) Oda I 2007 arXiv:0709.2419 [hep-th]

[9] Kakushadze Z 2008 Phys. Rev. D 77024001 (arXiv:0710.1061 [hep-th])

[10] Percacci R 1991 Nucl. Phys. B 353271 (arXiv:0712.3545 [hep-th])

[11] Chamseddine A H 2003 Phys. Lett. B 557247 (arXiv:hep-th/0301014)

[12] Kirsch I 2005 Phys. Rev. D 72024001 (arXiv:hep-th/0503024)

[13] Parthasarathy R 1986 Phys. Lett. B 18191 Leclerc M 2006 Ann. Phys. 321708 (arXiv:gr-qc/0502005)

[14] Omero C and Percacci R 1980 Nucl. Phys. B 165351 Gell-Mann M and Zwiebach B 1984 Phys. Lett. B 141333 Demir D A and Pulice B 2006 Phys. Lett. B 6381 (arXiv:hep-th/0605071)

[15] Van Nieuwenhuizen P 1973 Nucl. Phys. B 60478

[16] Rosen N 1940 Phys. Rev. 57147 Rosen N 1940 Phys. Rev. 57150 Moffat J W 2003 Int. J. Mod. Phys. D 12281 (arXiv:gr-qc/0202012) 